\title{
A Evolução do Pandorga GNU/Linux, Uma Distribuição para Uso no Ensino Fundamental
}

\author{
Rainer Krüger1 \\ 1Universidade Luterana do Brasil (Ulbra) - Curso de Ciência da Computação \\ Av. Farroupilha, 8.001 - Bairro Luís - CEP 92420-280 - Canoas - RS \\ junior_gdf@yahoo.com ${ }^{1}$
}

\begin{abstract}
This article describes the evolution of Pandorga GNU/Linux, a version for distribution on primary schools. The Pandorga project has presented a postive evolution since its beginning in july of 2006. New softwares have been created for the system and also a few mistakes have been corrected, not to mention improvements on its user environment.The main intention on this article is to present the improvements which are being made on the distribution of Pandorga GNU/Linux and also the impact the system may have on the quality of Brazilian education system and also on the free software campaign.
\end{abstract}

Keywords: Free Software; Open Source; GNU/Linux Distribuition; Education; Computing

Resumo. Este artigo descreve a evolução do Pandorga GNU/Linux, uma distribuição para uso no ensino fundamental. Desde seu início em Julho de 2006, o projeto vêm evoluindo e apresentando resultados satisfatórios com a adição de novos programas, correção de erros e melhorias de ambiente. O objetivo principal deste artigo é apresentar uma visão geral das melhorias que estão sendo realizadas na distribuição Pandorga GNU/Linux, e como elas podem refletir no aumento de qualidade da educação brasileira e do movimento do software livre.

Palavras-chaves: Software Livre; Open Source; Distribuição GNU/LINUX; Educação; Informática.

\section{Introdução}

O projeto Pandorga GNU/Linux [Pandorga 2006] teve início em Julho de 2006 a partir de uma parceria entre o autor e a Secretaria Municipal de Ensino e Pesquisa de Cachoeirinha, RS. O objetivo inicial era de pesquisar as necessidades computacionais dos laboratórios de informática do ensino fundamental e buscar uma distribuição sob a licença de Software Livre que substituísse o atual parque tecnológico. Constatou-se que das distribuições educativas avaliadas, nenhuma possuía todas as características necessárias, mas, a maioria das necessidades de programas educativos estavam disponíveis na Internet, alguns necessitando de adaptações e traduções. A proposta então foi desenvolver uma distribuição GNU/Linux que agregasse exatamente as necessidades computacionais do ensino fundamental com ambientes organizados e direcionados às crianças. Também deveria estar traduzido para o português e possuir uma interface que favorecesse o uso e adaptação dos educadores e alunos. O projeto envolvia também a criação de documentação voltada às crianças.

Primeiramente, uma pesquisa foi realizada com professores e demais funcionários ligados à Secretaria de Ensino e Pesquisa de Cachoeirinha, RS, identificando seus conhecimentos em informática e as necessidades de recursos de software durante as aulas para definir os programas que satisfizessem estas necessidades. Na área da Matemática as necessidades encontradas foram: cálculos, figuras e formas geométricas, gráficos, memória, números, raciocínio lógico e tabelas. Em Português: alfabeto, desenhos, digitação, histórias infantis, leitura e produção textual. Outras atividades: anatomia, blog, geografia, jogos, 
pesquisa/Internet e testes. $\mathrm{Na}$ ocasião, também foram definidas questões relacionadas a usabilidade e ergonomia do sistema.

A partir dos dados levantados, uma distribuição base foi pesquisada para desenvolver a distribuição educacional. Da distribuição base se aproveitaria o núcleo do sistema, kernel, servidores e ferramentas básicas para o funcionamento geral, gerenciador de janelas, programas de uso geral como navegadores de internet, ferramentas de escritório, ferramentas de configuração e instalador. A distribuição também deveria estar traduzida para o português e possuir uma interface gráfica com programas amigáveis e de fácil utilização.

A distribuição Kurumin Linux [Kurumin 2007] se mostrou ideal às necessidades além de ter êxito em relação à adaptação durante testes realizados com alunos e educadores em um dos laboratórios que futuramente receberiam a nova distribuição. Ela destina-se a usuários finais, possuindo programas e ferramentas que contemplam em sua maioria as necessidades comuns deste tipo de usuário, sendo que grande parte traduzidos para a língua portuguesa. Por ser desenvolvida no Brasil, ela também é compatível com o hardware utilizado aqui. Outra característica importante para este projeto é o fato desta distribuição poder ser executada diretamente a partir do $\mathrm{CD}($ Live - $C D)$. A versão inicial do Pandorga foi baseada na versão estável do Kurumin Linux, 6.1.

É relevante ressaltar que todos os programas usados no Kurumin Linux estão sob a licença de Software Livre GNU/GPL (GNU Public Licence). Plug-ins e codecs proprietários necessários para a correta utilização de alguns formatos de arquivo são oferecidos nos passos finais da instalação, respeitando as devidas licenças. Isto se torna útil à distribuição Pandorga por aumentar a compatibilidade e usabilidade da distribuição respeitando as licenças de cada desenvolvedor.

Em relação aos programas educacionais utilizados na distribuição, para sua seleção, foram realizados processos de pesquisa em cada área de conhecimento necessária. A partir dos programas encontrados, foram feitas análises buscando os candidatos que melhor contemplavam as necessidades.

$\mathrm{Na}$ área de matemática, os principais programas selecionados foram o CubeTest que trabalha o raciocínio lógico através destas formas, KMathTest que gera testes aritméticos e equações do primeiro grau e o TuxMath, um jogo matemático que tem por objetivo estimular o raciocínio lógico através cálculos para crianças. Também foi usado o GCompris, o OpenOffice e sites de internet com jogos e demais conteúdos sobre o tema contemplando o restante das necessidades encontradas.

Para as necessidades de português, os programas principais são KHangMan, um jogo da forca que estimula a associação do vocabulário e alfabeto, e o KTouch que é um programa para exercitar a digitação. As necessidades de digitação e leitura são contempladas pelo programa GCompris. Outros programas desta categoria, que contemplam a necessidade de histórias infantis, são encontrados em formato proprietário, mas com acesso através de sites da Internet. Para estes programas foram gerados ícones de acesso.

Buscando contemplar as necessidades restantes, foram inseridos programas multidisciplinares como GCompris, Open Office e TuxPaint. O GCompris é uma suíte educacional com um total de 80 programas, incluindo as áreas de matemática, português, ciências, geografia e jogos diversos. Ele é encontrado em muitas distribuições educativas, já recebendo prêmios internacionais. O OpenOffice é a suíte Office em Software Livre mais usada no mundo, sendo importante nas necessidades de cálculo, gráficos e tabelas para a área de matemática, produção textual e digitação para a área de português além do desenvolvimento de histórias infantis e criação de apresentações. O TuxPaint é um 
programa de desenho totalmente direcionado à crianças com ferramentas simples e criativas. Por fim, o KGeography foi inserido contemplando a necessidade de geografia, ele possui mapas de diversos países com a possibilidade de criar questionários sobre regiões, nomes de capitais e associação de bandeiras. A única necessidade não contemplada na primeira versão da distribuição foi a de anatomia, contemplada posteriormente com o programa Homem-Batata.

Durante o desenvolvimento da distribuição, alguns dos programas foram obtidos em código-fonte e compilados manualmente, seja por não haver a versão destes programas em pacote deb ou por ser necessário fazer alterações e/ou traduções no programa. Os pacotes deb foram então criados e disponibilizados aos interessados através de um repositório na Internet no endereço http://educacao.cachoeirinha.rs.gov.br/linux.

Após instalados os programas necessários, foi configurado o ambiante gráfico, telas de inicialização, arquivos do sistema, entre outras configurações. Foram criados três usuários no sistema, um administrativo, outro para as séries iniciais, de pré à $4^{\mathrm{a}} \mathrm{e}$ um para as séries finais, de $5^{\mathrm{a}}$ à $8^{\mathrm{a}}$. Cada ambiente foi personalizado de forma que a distribuição não fique dispersa para os alunos, levando em consideração a forma visual e a disponibilidade dos ícones e programas. A Figura 1 mostra a área de trabalho da distribuição Pandorga GNU/Linux 0.1:

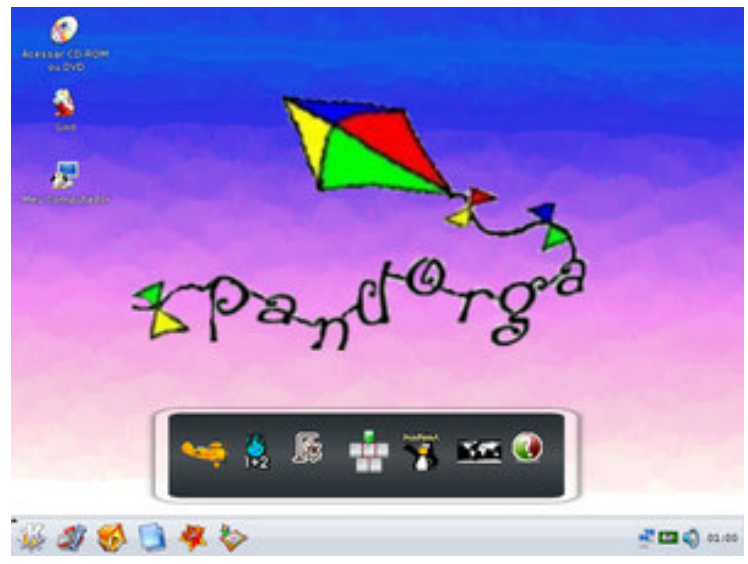

Figura 1. Área de trabalho da distribuição Pandorga GNU/Linux 0.1

Entre os objetivos do Pandorga GNU/Linux estavam a criação de um ambiente voltado a crianças, com uma linguagem específica para elas; e a criação de uma distribuição GNU/Linux que possa ser utilizada livremente. Assim, escolas que desejarem se beneficiar do uso de software livre em seus laboratórios terão uma distribuição com programas educativos. Em Dezembro de 2006 foi lançada a primeira versão, em testes, do Pandorga GNU/Linux, versão 0.6. Ela possuía grande parte das necessidades de software contemplada, um ambiente específico para as crianças e programas traduzidos em sua maioria para o português atingindo os resultados esperados. Porém o desenvolvimento de documentação não foi alcançado.

A distribuição foi batizada de Pandorga GNU/Linux, nome gaúcho para papagaio ou pipa, que simboliza a liberdade de vôo, ou, a liberdade de novos horizontes, novos conhecimentos e possibilidades através de uma brincadeira infantil.

Informações mais detalhadas sobre o desenvolvimento da distribuição e escolha dos programas educacionais podem ser encontrados no relatório final do trabalho de conclusão de curso de Krüger ([2006]). 
As próximas 3 seções abordam a atualização da distribuição Pandorga GNU/Linux: Os resultados alcançados na versão 0.5 , os projetos em desenvolvimento, avaliação e conclusão.

\section{Características da versão 0.5}

Com o lançamento do Kurumin Linux 7.0 em Fevereiro de 2007, o Pandorga GNU/Linux entrou em novo estágio de desenvolvimento, sendo baseado no Kurumin Linux Light 7.0. A versão Light do Kurumin possui uma quantidade menor de programas em relação à da distribuição principal, com configurações que exigem menos recursos de hardware mas preservando suas funcionalidades. Por ser uma distribuição voltada à educação, o Pandorga têm o objetivo de possuir somente programas necessários às escolas, tornando seu desenvolvimento mais interessante a partir do Kurumin Linux Light. Em meados de Fevereiro a versão 0.5 do Pandorga GNU/Linux foi iniciada.

Esta versão preservou os programas educacionais instalados na antiga versão adicionando dois novos programas: o Homem-Batata para as necessidades de anatomia infantil, onde é possível trabalhar as noções de corpo humano, e o Kalzium, um programa de química que possui uma tabela periódica completa e educativa. O conceito de ambientes para faixas de escolaridade também foi mantido, mas com novas configurações. A figura 2 mostra a área de trabalho da distribuição Pandorga GNU/Linux 0.5:

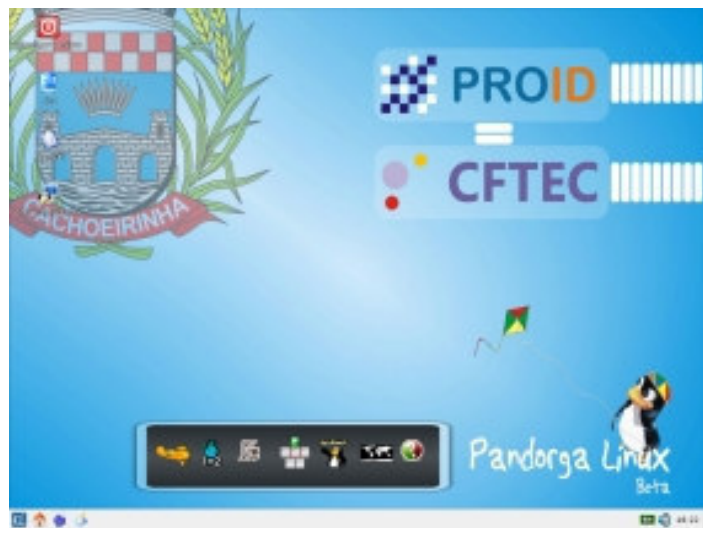

Figura 2. Área de trabalho da distribuição Pandorga GNU/Linux 0.5

Os programas já instalados foram refinados nas suas configurações, a partir de testes com a distribuição, pode-se ser mais preciso quanto as características de cada programa. Por exemplo, o KMathTest possui configurações diferentes nos ambientes de pré à $5^{\mathrm{a}}$ e de $6^{\mathrm{a}}$ à $8^{\mathrm{a}}$, os intervalos de números aplicados às questões e os tipos de equações são diferentes, adequados a cada faixa de ensino. Os demais programas também foram adequados às faixas de ensino. Durante o desenvolvimento desta versão, o instalador do Kurumin foi adaptado

ao Pandorga, recebendo novas informações durante o processo de instalação, configurações específicas aos usuários e pequenas alterações gráficas. A telas e alguns parâmetros de inicialização (boot) também foram modificados tornando o sistema mais personalizado.

Por fim, erros encontrados na primeira versão foram corrigidos em sua maioria, tornando sistema mais estável. O projeto agora melhor testado e melhor direcionado às crianças teve sua versão lançada em Abril de 2007.

\section{Projetos em desenvolvimento}

Esta seção contém informações sobre o processo atual de desenvolvimento da distribuição e projetos que devem ser realizados em seguida. São alterações relevantes ao 
sistema tornando-o mais funcional e completo. Todas as alterações estarão disponíveis para que outros projetos e distribuições possam se beneficiar, respeitando a licença GNU/GPL e fomentando a colaboração dentro do movimento Software Livre e educacional.

\subsection{Ferramentas administrativas}

Continuando as alterações da versão 0.5 , o instalador gráfico será personalizado para o Pandorga. Baseado no instalador do Kurumin disponível na versão 7.0, o instalador do Pandorga já é adaptado para criar os usuários utilizados na distribuição, agora, a distribuição deve ter maior possibilidade de configuração para os mesmos, permitindo alterar seu ambiente como plano de fundo, arquivos básicos e disponibilidade de programas no menu.

Sua interface gráfica será adaptada à distribuição Pandorga sendo alterada também as mensagens enviadas durante a instalação, pretende-se dar explicações básicas sobre os programas educacionais existentes durante o processo de instalação. Isto será interessante aos usuários que pretendem instalar a distribuição em sua casa. Já aos administradores dos laboratórios de escola, pretende-se criar um sistema de instalador automático, a partir de um arquivo pré-configurado, o sistema é instalado automaticamente sem a necessidade de intervenção do administrador. Esta opção será especialmente interessante para agilizar a instalação em locais com uma grande quantidade de computadores.

O Kurumin também disponibiliza o Clica-aki, esta é uma central de controle do sistema onde é possível realizar configurações diversas, manutenção dos principais programas instalados e configuração de ambiente. Duas grandes configurações devem ser realizadas neste painel: a área de manutenção dos programas será adaptada para programas educacionais; e o painel será modificado para que cada tipo de usuário possa realizar somente configurações que lhes diz respeito. Atualmente o Clica-aki permite que somente o administrador, ou usuário que tenham tal permissão, realize configurações neste sistema. Para o Pandorga, é interessante que o administrador, ou o usuário com tal permissão, realize as configurações do sistema e manutenção dos programas e, os usuários "alunos" realizem determinadas configurações em seu ambiente. Esta opção também deverá ser informada durante a instalação, pois em certos casos, convém a restrição total dos usuários em relação às suas configurações.

\subsection{Pacotes Deb}

Os pacotes criados para o Pandorga com os programas educativos serão atualizados sob uma nova perspectiva: O programa ficará num pacote mais genérico e as configurações de ambientes estarão em outro pacote, totalmente adaptadas ao Pandorga. Isto permitirá que um usuário de outra distribuição possa usar os programas desenvolvidos pelo projeto sem problemas. Esta atualização permitirá também a configuração de programas não mantidos pelo Pandorga, até a versão 0.5 , as configurações eram realizadas depois do programa instalado, sem a criação de um pacote para isto.

As adaptações ou traduções realizadas em projetos não pertencentes ao Pandorga estão recebendo suas atualizações, ou seja, a exemplo do Kgeography, que foi traduzido para o português durante o desenvolvimento do Pandorga, o projeto original esta recebendo esta contribuição, sendo possível sua disponibilidade nas próximas versões do programa. Isto é vital para o bom funcionamento do Software Livre, as colaborações devem retornar ao projeto principal para que o mesmo evolua.

Um meta-pacote, pacote que gerencia a instalação de outros pacotes, será criado para o Pandorga. Este meta-pacote requisitará a instalação, atualização ou remoção dos pacotes com os programas e configurações do Pandorga, isto permitirá a instalação da distribuição em qualquer outra distribuição baseada no Debian. Assim, se um usuário que já 
utiliza o Linux pretender adicionar as funcionalidades do Pandorga em seu sistema, ele irá instalar este meta-pacote e todos os programas educacionais bem como as devidas configurações serão automaticamente instaladas. Este meta-pacote permitirá que o projeto Pandorga se una à outros projetos educacionais, fortalecendo seus vínculos e ampliando seu desenvolvimento e utilização.

\subsection{Traduções e documentação}

A tradução, adaptação e desenvolvimento de novos programas ou dos programas já utilizados é uma das principais linhas de desenvolvimento do Pandorga. Todos os programas utilizados na distribuição devem estar devidamente traduzidos para a língua portuguesa, em bom funcionamento e atendendo às necessidades pedagógicas. Um das características do Pandorga GNU/Linux é de desenvolver uma distribuição baseada nas necessidades pedagógicas e não o contrário. Novos programas devem ser desenvolvidos de acordo com as necessidades identificadas em Cachoeirinha ou outras escolas e cidades. Para isto uma grande comunidade deve ser criada envolvendo educadores, tradutores, desenvolvedores, testadores e avaliadores, em várias instâncias da educação.

O Pandorga têm como objetivo paralelo ao desenvolvimento da distribuição a criação de documentação para os educadores e alunos. Ciente da necessidade de documentação específica aos usuários, o projeto Pandorga pretende criar documentação voltada às crianças com linguagem adequada, possibilitando a boa utilização do sistema.

É prevista a criação de cartilhas com desenhos infantis abordando assuntos relacionados ao Linux e aos programas contidos no Pandorga.

Também é pretendida a tradução da documentação dos programas usados na distribuição que ainda não foram traduzidos, este tipo de documentação será útil aos educadores que buscam um conhecimento aprofundado do programa e a comunidade Software Livre em geral.

\subsection{Ambiente}

Atualmente o Pandorga GNU/Linux utiliza como gerenciador de janelas gráficas o $\mathrm{KDE}$, este, apesar de consumir muitos recursos de hardware, é um dos gerenciadores mais usados no Linux por ser muito completo e funcional. Ele também se assemelha ao ambiente gráfico do Microsoft Windows ${ }^{\circledR}$, facilitando a adaptação dos novos usuário no Linux. Em computadores que possuem bons recursos de máquina, ele se torna ideal, porém os computadores mais antigos necessitam de um gerenciador de janelas mais simples, com menos consumo de recursos.

Assim, é prevista a instalação do gerenciador de janelas TDE, que se assemelha ao KDE mas possui menos recursos diminuindo a carga no sistema. Para que este novo gerenciador de janelas esteja totalmente funcional, é necessário a criação dos ambientes dos usuários, testar todos os programas e avaliar se realmente tornou-se funcional o sistema em computadores com menos recursos.

\section{Conclusão}

No artigo foram apresentadas as características do Pandorga GNU/Linux, sua evolução e os projetos em desenvolvimento. Como objetivo geral, buscou-se explanar sobre os projetos em desenvolvimento, mostrando seu potencial e como eles podem colaborar com a educação brasileira e o movimento Software Livre.

O Pandorga têm importante papel no desenvolvimento da qualidade educacional brasileira permitindo que educadores e crianças utilizem um sistema livre, de qualidade e gratuito em suas escolas de ensino fundamental, fomentando conceitos filosóficos como colaboração, liberdade, moral, respeito às autorias e sustentabilidade. 
Para o movimento do Software Livre, o Pandorga é mais uma opção de distribuição que colabora com o desenvolvimento de aplicações livres auxiliando em traduções, adaptações, documentação e testes. O Pandorga têm especial importância na disseminação do Software Livre por ser utilizado por crianças com talvez pouco ou nenhum conhecimento em informática. Assim, estas crianças já iniciam seus conhecimentos digitais tendo liberdade de escolha no meio digital e social.

\section{Referências}

Kurumin, Kurumin Linux. 2007 Disponível em:

<http://www.guiadohardware.net/gdhpress/kurumin/> Acesso em: 06 jun. 2007.

KRÜGER, Rainer. Desenvolvimento de uma Distribuição GNU/Linux para Auxílio à Educação Fundamental. [Dez. 2006]. 25 p. Orientador: Roland Teodorowitsch.

Monografia (Bacharelado) - Curso de Ciência da Computação, Universidade Luterana do Brasil, Canoas. [2006].

Pandorga, Pandorga GNU/Linux. 2006 Disponível em:

<http://educacao.cachoeirinha.rs.gov.br/linux> Acesso em: 02 jun. 2007. 